\title{
Light chain deposition disease presenting with hepatomegaly: an association with amyloid-like fibrils
}

\author{
G. Pelletier, ${ }^{1}$ M. Fabre, ${ }^{2}$ P. Attali, ${ }^{1}$ A. Ladouch-Badre, ${ }^{2}$ O. Ink, ${ }^{1}$ E. Martin ${ }^{2}$ \\ and J.-P. Etienne ${ }^{1}$
}

Departments of ${ }^{1}$ Gastroenterology and ${ }^{2}$ Pathology, Bicêtre Hospital, Le Kremlin Bicêtre, France.

\begin{abstract}
Summary: We report an unusual case of lambda light chain deposits without overt plasma cell dyscrasia. The clinical presentation was hepatomegaly without biochemical sign of renal involvement. Portal hypertension, spontaneous rupture of the spleen and fracture of the 12th thoracic vertebra occurred during the course of the disease. Ultrastructural studies showed that lambda light chain deposits were associated with amyloid-like deposits. This case suggests that light chain deposition disease and amyloidosis could be two expressions of the same disease.
\end{abstract}

\section{Introduction}

Since Randall et al. ${ }^{1}$ described the light chain deposition disease (LCDD) which is a systemic disease with constant and predominant renal involvement, numerous cases of glomerulonephritis with light chain deposits have been reported. We report here a case of LCDD unusual in that the clinical presentation was hepatomegaly without biochemical evidence of renal involvement and ultrastructural studies showed that light chain deposits were associated with amyloid-like deposits.

\section{Case report}

A 63 year old woman was referred in March 1984 for epigastric pain and a $10 \mathrm{~kg}$ weight loss. On physical examination a $15 \mathrm{~cm}$ large, regular and firm hepatomegaly was palpated. Total serum bilirubin was $44 \mu \mathrm{mol} / 1$, serum alkaline phosphatase 469 IU/1 (normal 30-80), serum aspartate aminotransferase $47 \mathrm{IU} / 1$ (normal 5-30). Prothrombin time (expressed as per cent of normal) was $75 \%$, serum albumin $35 \mathrm{~g} / \mathrm{l}$ and serum creatinine $71 \mu \mathrm{mol} / 1$. Proteinuria and microscopic haematuria were absent. An abdominal ultrasound examination

Correspondence: G. Pelletier, M.D., Service des Maladies du Foie et de l'Appareil Digestif, Hôpital de Bicêtre, 78 rue du Général Leclerc, 94275 Le Kremlin Bicêtre Cedex, France.

Accepted: 11 May 1988. showed homogeneous hepatomegaly; the kidneys appeared normal. At laparoscopy the liver was regular, pale and enlarged. A liver biopsy was performed and LCDD was diagnosed on the presence of an amorphous material negative for amyloid stainings (Congo red, thioflavine $\mathrm{T}$ ) but positive for anti-lambda light chain staining (Figures 1 and 2). It was distributed along the spaces of Disse and the centrilobular and portal areas and outlined the sinusoids and laminated the hepatocytic plates (Figure 1). There was no peliosis. Bone marrow aspirate, serum and urine immunoelectrophoresis were normal. A bone marrow biopsy was performed (see pathological findings). Skeletal Xrays just showed an axial demineralization. The patient received cyclophosphamide, melphalan and prednisolone from April to October 1984. In June 1984, spontaneous rupture of the spleen occurred requiring splenectomy. Bone marrow aspirate, serum and urine immunoelectrophoresis were normal. The spleen contained multiple cystic nodules $1-15 \mathrm{~mm}$ in diameter filled with red cells and fibrin and surrounded by amorphous material.

The patient was again hospitalized in November 1984 because of sudden thoracic pain with weakness of the legs. Skeletal X-rays showed diffuse demineralization with collapse of the 12th thoracic vertebra; no other lesion could be detected. Bone marrow aspirate, urine and serum immunoelectrophoresis were still normal. Laminectomy and a stabilization of the thoracolumbar spine by a plate

(C) The Fellowship of Postgraduate Medicine, 1988 


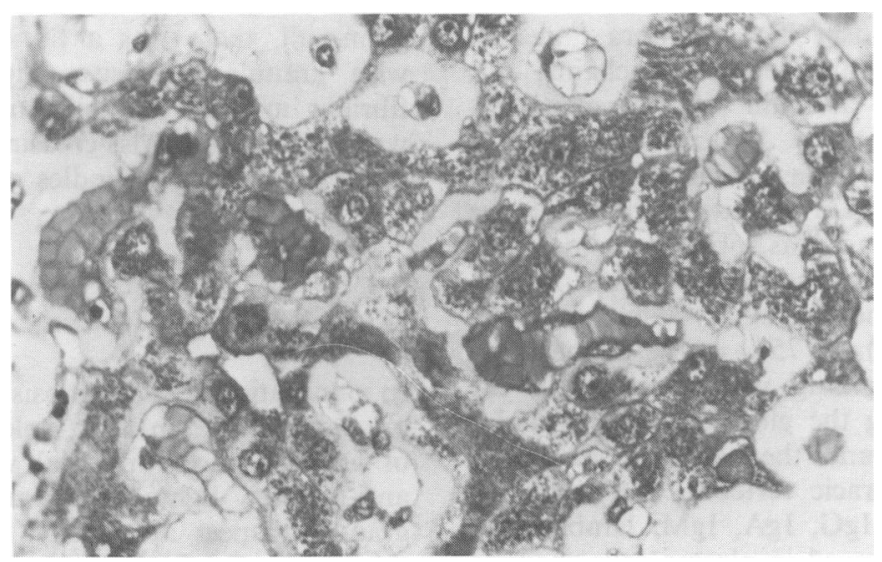

Figure 1 Liver biopsy shows an amorphous material along the spaces of Disse, which laminates the hepatocytic plates. Trichrome stain, magnification $\times 672$.

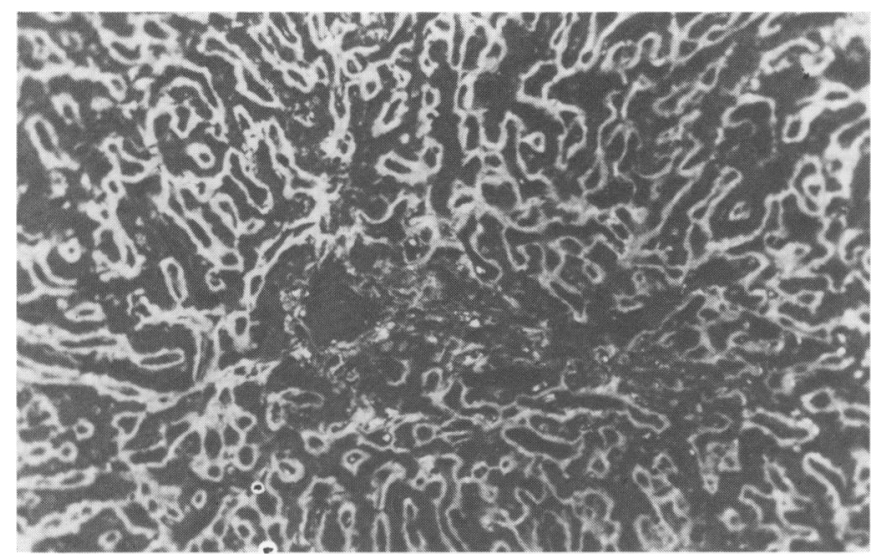

Figure 2 Immunofluorescence study of the liver: anti-lambda light chain gives a positive staining along the sinusoidal spaces. Magnification $\times 420$.

were performed. The vertebral body was destroyed by a diffuse amorphous material which extended into the paravertebral muscles and ligaments.

She was readmitted on 24 December 1984 for encephalopathy. Physical examination showed asterixis, jaundice, ascites and oedema of the legs. Ascitic protein concentration was $16 \mathrm{~g} / \mathrm{l}$. Serum bilirubin was $398 \mu \mathrm{mol} / 1$ and serum creatinine $153 \mu \mathrm{mol} / 1$. There was still no proteinuria. Bone marrow aspirate, serum and urine immunoelectrophoresis were normal. Ultrasound examination showed recanalization of the umbilical vein. The patient died in coma one month after admission. An autopsy was performed.
In $15 \%$ of the glomeruli there was moderate mesangial proliferation, and amorphous material was visible in the interstitium and in some capillary and tubular walls. In the liver the sinusoidal deposits appeared more abundant than in March 1984, and hepatocytic plates were considerably laminated.

In the liver, bone marrow, spleen and thoracic vertebrae, the deposits were Congo red-negative and showed no birefringence under cross-polarization. Thioflavine $\mathrm{T}$ staining was negative. Known amyloid liver sections were used as controls.

Direct immunofluorescence was performed on hepatic and renal frozen specimens from biopsy 
(liver) and autopsy (liver and kidneys). Commercial fluoresceinated monospecific antisera against lambda and kappa immunoglobulin chains, C3, Clq, C4, fibrin and albumin were used (Behringwerke .and Sebia Dako). Positive and negative kidney specimens served as controls. Only antilambda light chain gave a positive staining. In the liver, a strong staining was observed along the sinusoidal spaces and in the vascular walls, in the interstitium and around the biliary ductules of the portal areas (Figure 2). In $75 \%$ of the glomeruli, a bright and coarse staining was observed in the mesangial areas, along the glomerular and tubular basement membrane and the capillary walls. On bone marrow and thoracic vertebral body sections, positive stainings for IgG, IgA, IgM, lambda and kappa chain were observed in plasma cells showing a polyclonal immunoglobulin secretion.

Electron microscopy was performed on material from biopsy (liver) and autopsy (liver and kidneys). In both liver and renal specimens, ultrastructural study showed the presence of finely granular electron-dense basement-like material which appeared nearly black. In the liver, this material was essentially located in enlarged Disse spaces which were filled by irregular clumps of granular substance (Figure 3). The deposit was also observed in the portal areas, along the collagen fibrils, the basement membrane of biliary ductules and in the vascular walls. The glomeruli showed prominent nodular mesangial areas. There was striking diffuse infiltration of both mesangial matrix and peripheral capillary basement membranes by the dense granular deposit (Figures 4 and 5). Some granular deposits were also observed in tubular basement membranes, interstitium and blood vessels. In some glomeruli, there was a fibrillar deposit associated with granular deposits (Figures 4 and 5). The fibrillar material was similar to amyloid fibrils: it was formed by criss-crossing, 9 to $10 \mathrm{~nm}$ thick, randomly oriented bundles of fibrils (Figure 5).

\section{Discussion}

In our patient the diagnosis of LCDD was based on the presence in liver, spleen, bone and kidneys of an amorphous material which reacted with an anti-lambda light chain antiserum and had a granular aspect at electron microscopy. However, this case of LCDD was unusual because of its clinical presentation and the association of amyloidlike and light chain deposits.

Renal involvement is observed in all cases of LCDD and almost always reveals the disease. ${ }^{1-3}$ In our patient, LCDD was discovered by hepatomegaly when there was no biochemical sign of renal involvement. To our knowledge, only 2 other cases of LCDD have presented with hepatomegaly ${ }^{4,5}$ but proteinuria was present at the time of diagnosis. In our patient post-mortem microscopic study showed some renal deposits, but renal failure only appeared 3 weeks before death. Portal hypertension occurred during the course of the disease as attested by recanalization of the umbilical vein and the presence of ascites with low protein concentration. Portal hypertension is uncommon in LCDD, reported in only 2 other cases. ${ }^{2,4}$ It might be explained by the presence of abundant deposits in

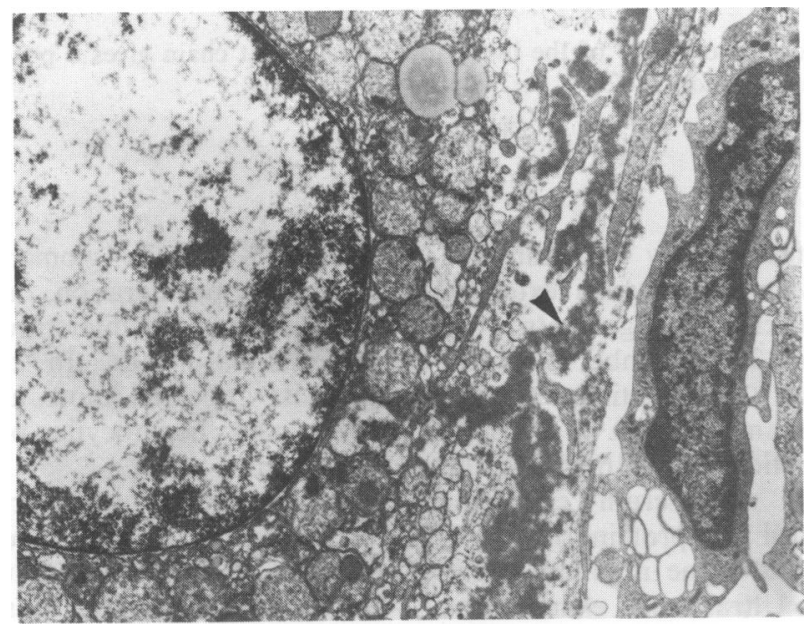

Figure 3 Liver biopsy - an electron photomicrograph shows the presence of abundant granular dense deposits in Disse space (arrow). Magnification $\times 6790$. 


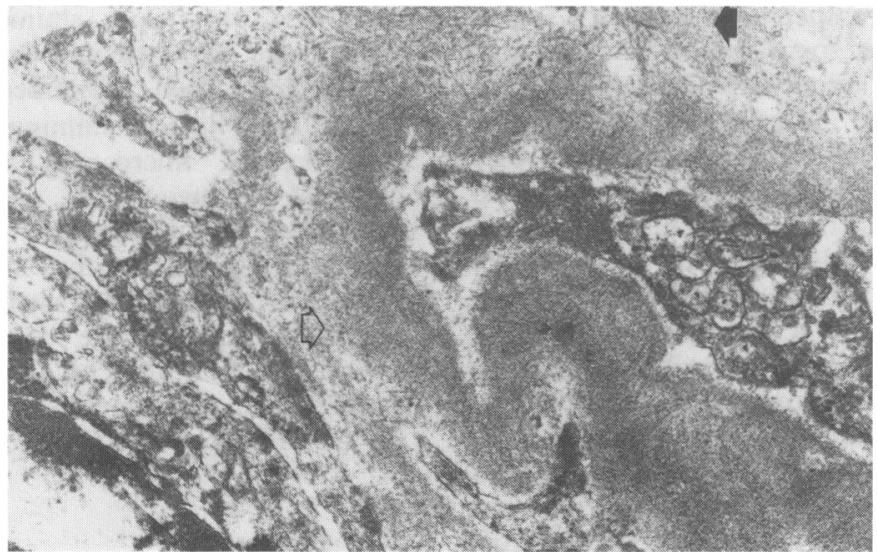

Figure 4 Renal biopsy - an electron photomicrograph shows the presence of granular (lighter arrow) and fibrillar (darker arrow) deposits. Magnification $\times 18,200$.

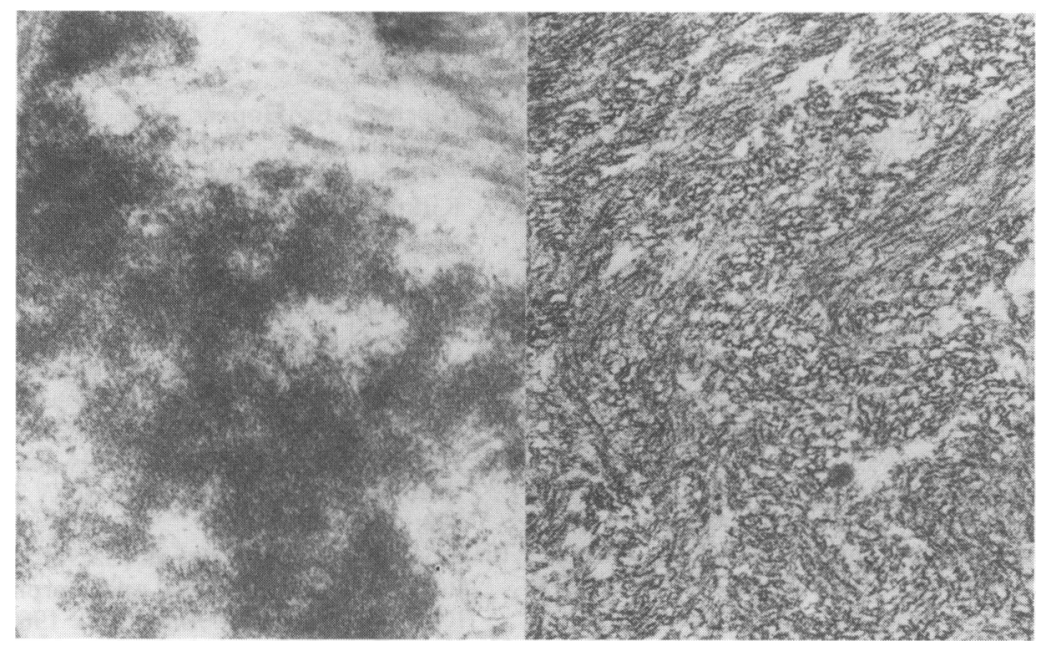

Figure 5 Renal biopsy - closer view of electron-dense granular deposit (left) and of randomly oriented microfibrils (right). Magnification $\times 56,000$.

the Disse spaces which caused sinusoidal obstruction of the portal venous system.

In our case, two unusual complications appeared during the course of the disease: spontaneous rupture of the spleen and fracture of the 12th thoracic vertebra producing spinal cord compression. Such complications have not been reported previously, even though spleen and bone involvement have been described. ${ }^{1,2,5}$ The appearance of peliosis in the spleen was the same as that observed in the liver among some patients with LCDD. ${ }^{2,3,5}$
Another peculiarity of our patient was the ultrastructural aspect: fibrillar amyloid-like deposits were associated with the granular deposits usually described in LCDD (Figures 4,5). To our knowledge there are only four reported cases of such an association: in one case of LCDD reported by Hoffman-Guilaine et al., ${ }^{5}$ ultrastructural study showed fibrillar and granular deposits in the walls of renal small vessels. Alpers et al. ${ }^{6}$ described a polyclonal light chain glomerulopathy with amyloid-like deposits. Recently Kirkpatrick et al. ${ }^{7}$ 
reported a case of multiple myeloma with kappa light chain deposits in some organs and amyloid deposits in others, the kidney was the only organ to show both amyloid and light chain deposits. Smith and Malcolm ${ }^{8}$ however, found in a patient with myeloma, amyloid deposits in hepatic arteries and lambda light chain deposits in the sinusoidal spaces.

In fact, although ultrastructural aspects of LCDD and amyloid deposits are quite different, there are some similarities between LCDD and ALtype amyloidosis: the amyloid-fibril protein is a homogeneous intact immunoglobulin light polypeptide chain whose cellular source is probably an immunocyte-derived clone. ${ }^{9}$ So, in our patient, light chain and amyloid deposit could be two different expressions of the same immunocyte derived abnormality in protein synthesis. Clinical and biological signs of liver involvement observed in amyloidosis and LCDD were similar. ${ }^{10,11}$ Using light micros-

\section{References}

1. Randall, R.E., Williamson, W.C., Mullinax, F., Tung, M.Y. \& Still, W.J.S. Manifestations of systemic light chain deposition. Am J Med 1976, 60: 293-299.

2. Ganeval, D., Mignon, F. Preud'Homme, J.L. et al. Dépot de chaines légères et d'immunoglobulines monoclonales: aspects néphrologiques et hypothèses physiopathologiques. In: Flammarion (ed) Act Nephrol Hop Necker. Paris 1981, 179-214.

3. Droz, D., Noel, L.H., Carnot, F., Degos, F., Ganeval, D. \& Grunfeld, J.P. Liver involvement in nonamyloid light chain deposits disease. Lab Invest 1984, 50: 683-689.

4. Mignon, F., Morel-Maroger, L., Cerf, M., Preud'Homme, J.L. \& Richet, G. Depots hépatiques et rénaux de chaines légères kappa révélateurs d'une dysglobulinémie. Nephrologie 1980, 1: 167-170.

5. Hoffman-Guilaine, C., Nochy, D., Tricottet, V., Mallet, L., Bariety, J. \& Camilleri, J.P. La maladie des dépots de chaines légères: une entité anatomoclinique. Ann Pathol 1984, 4: 105-113. copy and the usual'stainings, histological liver lesions are also similar, except peliosis hepatis which has been described in few cases of LCDD. ${ }^{2,3,5}$ Special stainings, immunofluorescence and electron microscopy make the distinction between the two kinds of deposits: light chain deposits are not birefingent when stained by Congo red, and give a positive staining with anti-light chain antiserum. Ultrastructural study shows granular electron-dense deposits in LCDD, and typical fine, non-branching and rigid fibrils in amyloidosis. Our case emphasizes the difficulty in classifying the deposition diseases.

\section{Acknowledgments}

The authors thank Nina Bandou and Catherine Corbet for secretarial assistance.

6. Alpers, C.E., Hopper, J. \& Biava, C.G. Light-chain glomerulopathy with amyloid-like deposits. Hum Pathol 1984, 15: 444-448.

7. Kirkpatrick, C.J., Curry, A., Galle, J. \& Melzner, I. Systemic kappa light chain deposition and amyloido sis in multiple myeloma: novel morphological obser-? vations. Histopathology 1986, 10: 1065-1076.

8. Smith, N.M.M. \& Malcolm, A.J. Simultaneous ALtype amyloid and light chain deposit disease in a liver biopsy: a case report. Histopathology 1986, 10: 1057-1064.

9. Glenner, G.G. Amyloid deposits and amyloidosis. The B-fibrilloses (first of two parts). $N$ Engl J Med 1980, 302: 1283-1292.

10. Levine, R.A., Amyloid disease of the liver. Correlation of clinical functional and morphologic features in forty-seven patients. Am J Med 1962, 33: 349-357.

11. Kyle, R.A. \& Bayrd, E.D. Amyloidosis: review of 236 cases. Medicine 1975, 54: 271-299. 